\title{
Modelling and Estimation of Carrier Frequency and Phase Uncertainties in Large Aperture Arrays
}

\author{
Akinbiyi Akindoyin \\ Electrical and Electronics Engineering, \\ Imperial College London \\ akinbiyi.akindoyin11@imperial.ac.uk
}

\begin{abstract}
This paper is concerned with the modelling and estimation of frequency and phase carrier uncertainties in an array system where its elements are distributed over a large area and consequently forming large aperture arrays with massive inter-element spacings. In this situation, it is practically impossible to have a common carrier (common local oscillator - LO) in order to form a fully coherent array system, as in the case of small aperture arrays where the array elements are in the vicinity of each other. Instead, each of the receiving array elements has its own independent carrier which will have slightly different frequencies and phases, even if the local oscillators are using similar hardware or even if each local oscillator is using a GPS reference clock for locking. These discrepancies will introduce frequency and phase uncertainties in the array which must be removed to achieve a coherent array system. In this paper, these uncertainties are mathematically modelled, their effect on the performance of an array system is examined and a simple method is proposed to estimate and eliminate these uncertainties. This work is supported by computer simulation studies and experimental results using a software defined radio (SDR) array testbed.
\end{abstract}

Index Terms-Frequency and phase uncertainties, array calibration, software defined radio, spherical wave propagation

\section{NOTATION}

A, a Scalar

$\underline{A}, \underline{a} \quad$ Column vector

A Matrix

$(\cdot)^{T} \quad$ Transpose

$(.)^{H} \quad$ Hermitian transpose

$(\cdot)^{*} \quad$ Conjugate

$\odot \quad$ Hadamard product

Hadamard division

$\|\underline{A}\| \quad$ Euclidian norm of the vector $A$

$\mathbb{A}^{\#} \quad$ Pseudo inverse of the matrix $\mathbb{A}$

$\mathbb{I}_{N} \quad N \times N$ Identity matrix

$\underline{0}_{N} \quad$ Column vector of $N$ zeros

$\underline{1}_{N} \quad$ Column vector of $N$ ones

$\mathcal{R}, \mathcal{C} \quad$ Set of real or complex numbers

$\measuredangle \quad$ element by element angle of a vector

$\mathcal{L}[\mathbb{A}] \quad$ Linear subspace spanned by the columns of $\mathbb{A}$

\section{INTRODUCTION}

The presence of uncertainties/errors in an array system of $N$ sensors causes rapid degradation in the performance of the system including its detection, estimation and resolution capabilities. For example, the effect of sensor location uncertainties on superresolution direction finding algorithm was presented in [1], [2]. Therefore, the array system should be calibrated to achieve its full capabilities. Array uncertainties can broadly be classified into electrical and geometrical uncertainties. Geometrical uncertainties arise due to the location of the array elements being imprecisely known while electrical uncertainties occur due to the imperfection in the electronics of the array system [1], for instance, inaccuracy in the gain and phase associated with the array elements [3]. Local oscillator (LO) uncertainties (consisting of carrier frequency and phase uncertainties) are form of electrical uncertainties that arise due to the fact that each of the array elements has its own carrier frequency which may be imprecisely locked to a common reference clock. Carrier frequency uncertainties will occur as a result of tuning errors in spite of a common reference signal applied to each array element while phase uncertainties arise due to the lack of a phase reference between the local oscillators. Array processing algorithms such as MUSIC and beamforming have the potential to be very powerful but at the same time are sensitive to these uncertainties. Hence, a well calibrated array system is therefore crucial to harness its full capabilities. Array calibration approaches in literature are largely based on collecting data from the array in the presence of external sources. The two main calibration techniques are pilot and self calibration. In pilot calibration, one or more sources with known parameters (e.g. location/direction) are used to estimate the array uncertainties. Examples can be found in [4], [5] and [6]. Additional techniques in literature include those considering moving pilot sources [7] and multifrequency pilot sources [1]. Self calibration approaches estimate at the same time both the array uncertainties and the source parameters [8] [9]. For example in [9], an objective cost function based on conditional maximum likelihood was proposed.

Nevertheless, the existing calibration methods assume a small aperture array with small intersensor spacing (order of one half-wavelength) and do not consider the carrier frequency and phase uncertainties between the array channels. However, 
in this paper, a large aperture array system is considered where the array elements are located several kilometers (say) from each other. This configuration makes it impractical for the array elements to share a common carrier but each element has its own carrier. This introduces additional frequency (tuning) errors and phase errors between the array elements and these errors prevent the array from operating as a fully coherent system. Furthermore, since the array elements are widely distributed, the plane wave propagation model is no longer valid. Hence, the spherical wave propagation model holds in this scenario. It is important to point out that arrays with widely distributed elements have a number of interesting applications areas including radar, geolocation and electronic surveillance.

The remainder of this paper is organised as follows: In Section II, a signal model of an array system with common local oscillator is presented. In Section III, the signal model for the large aperture array with independent LO is presented. Furthermore, the effects of the carrier frequency and phase uncertainties on the array system are illustrated with a representative example. In Section IV, a system structure is proposed for estimating and removing these uncertainties, making the array a coherent system. Computer simulations and experiments results using a Software Defined Radio (SDR) array testbed are presented in Sections V and VI. Finally, the paper is concluded in Section VII.

\section{Signal Modelling for Coherent ARrays}

Consider an array of $N$ omnidirectional elements with array geometry described by the matrix $\mathbf{r} \in \mathcal{R}^{3 \times N}$ such that,

$$
\mathbf{r}=\left[\underline{r}_{1}, \underline{r}_{2}, \cdots, \underline{r}_{N}\right]=\left[\underline{r}_{x}, \underline{r}_{y}, \underline{r}_{z}\right]^{T}
$$

where $\underline{r}_{i} \in \mathcal{R}^{3 \times 1}$ for $i=1,2, \cdots, N$ denotes the Cartesian coordinates of the $i^{t h}$ element in the array and $\underline{r}_{x}, \underline{r}_{y}, \underline{r}_{z}$ denote $N \times 1$ vectors of real numbers describing the $x, y$ and $z$ coordinates of the array elements respectively. Furthermore, without any loss of generality, the array elements are assumed isotropic. The wireless single input multiple output (SIMO) channel modelling for a coherent array system is shown in Figure 1. With reference to this figure, the transmitter transmits a baseband message signal $m(t)$ (see point A0) using the carrier $\exp \left(j 2 \pi F_{c} t\right)$ (see point A1). Assuming that the transmitter is located at $\underline{r}_{m} \in \mathcal{R}^{3}$ relative to the array reference point $(0,0,0)$ and ignoring multipaths (for notational convenience), the transmitted signal at point A2 arrives via a single line-of-sight (LOS) path at the input of the array (point A7) as a vector signal of $N$ elements.

It is clear from Figure 1 that the path from the transmitter to the array is modelled by a complex number $\beta$ (point A3) which represents the path gain from the transmitter to the array reference point ${ }^{1}$ and the array manifold vector $\underline{S}\left(\left\|\underline{r}_{m}\right\|, \theta_{m}, \phi_{m}\right) \in \mathcal{C}^{N \times 1}$ (point A4) which is the Rxarray response vector. The system operates in the presence

\footnotetext{
${ }^{1}$ It is common practice that $\beta$ also includes the power of the transmitter
} which allows the assumption that the Tx-signal $m(t)$ has unity power.

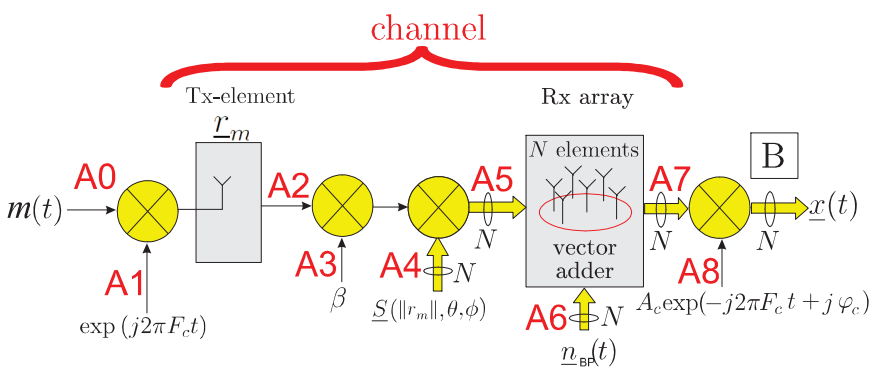

Fig. 1. Wireless Single Input Multiple Output (SIMO) Single Path Mode.

of additive white Gaussian bandpass noise $\underline{n}_{B P}(t) \in \mathcal{C}^{N \times 1}$ (see point A6) of zero mean (i.e. $\underline{0}_{N}$ ) and covariance matrix $\sigma_{n}^{2} \mathbb{I}_{N}$. A carrier at point A8 downconverts the bandpass signals at point $\mathrm{A} 7$ to the baseband signal $\underline{x}(t) \in \mathcal{C}^{N \times 1}$ at point B. As there is only a single carrier at point A8 and there are $N$ incoming signals at point $\mathrm{A} 7$, the amplitude $A_{c}$ and phase $\varphi_{c}$ are electronically adjusted to be of unity magnitude and in phase with the carrier received at the reference sensor (reference point ${ }^{2}$ ). This gives the signal vector $\underline{x}(t)$ at point B modelled as

$$
\underline{x}(t)=\underline{S} \cdot m(t)+\underline{n}(t)
$$

where vector $\underline{S} \triangleq \underline{S}\left(\theta_{m}, \phi_{m}, \underline{\rho}_{m}\right)$ represents the $N$ dimensional array manifold vector defined as follows:

$\underline{S} \triangleq\left(\left\|\underline{r}_{m}\right\|^{a} \cdot \underline{\rho}_{m}^{-a}\right) \odot \exp \left(-j \frac{2 \pi F_{c}}{c}\left(\left\|\underline{r}_{m}\right\| \cdot \underline{1}_{N}-\underline{\rho}_{m}\right)\right) \in \mathcal{C}^{N}$

where

$\underline{\rho}_{m}=\sqrt{\left\|\underline{r}_{m}\right\|^{2} \cdot \underline{1}_{N}+\underline{r}_{x}^{2}+\underline{r}_{y}^{2}+\underline{r}_{z}^{2}-\frac{\left\|\underline{r}_{m}\right\| c}{\pi F_{c}} \mathbf{r}^{T} \underline{k}\left(\theta_{m}, \phi_{m}\right)}$

with $a$ representing the path loss exponent, $c$ denoting the speed of light and $\underline{k}\left(\theta_{m}, \phi_{m}\right) \in \mathcal{R}^{3}$ denotes the wavenumber vector given by

$$
\underline{k}\left(\theta_{m}, \phi_{m}\right)=\frac{2 \pi F_{c}}{c} \underbrace{\left[\begin{array}{c}
\cos \theta_{m} \cos \phi_{m} \\
\sin \theta_{m} \cos \phi_{m} \\
\sin \phi_{m}
\end{array}\right]}_{\triangleq \underline{u}\left(\theta_{m}, \phi_{m}\right)}
$$

with $\underline{u}\left(\theta_{m}, \phi_{m}\right) \in \mathcal{R}^{3}$ representing the unity norm vector pointing in the direction of the transmitting source.

Note that the baseband noise $\underline{n}(t)$ now is of zero mean and covariance matrix $\frac{\sigma_{n}^{2}}{\beta} \mathbb{I}_{N}$. Thus, the SNR at point $\mathrm{B}$ is equal to the SNR at point A7.

\section{Signal Modelling for ARrays with CARrier UNCERTAINTIES}

In a scenario where an Rx-array of $N$ sensors does not have a common downconverter ${ }^{3}$ (i.e. independent carriers are

${ }^{2}$ It is also common to set one of the array elements as the reference point i.e. the "master" with the other array elements setting themselves as "slaves" with respect to this.

${ }^{3} \mathrm{~A}$ carrier on the receiver operates as a "downconverter". 
deployed), additional steps must be taken to allow the array to operate as a fully coherent system. Figure 2 illustrates the updated block diagram from point A5 in Figure 1 in the case that independent LOs are deployed.

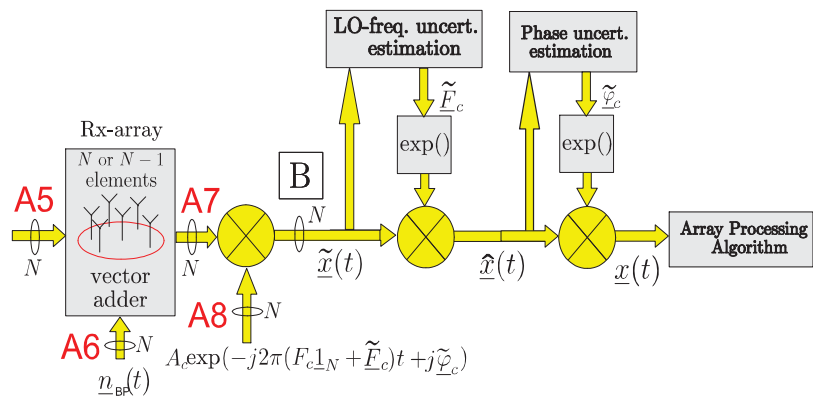

Fig. 2. Model of an array receiver with local oscillator uncertainties.

In particular, at point $\mathrm{A} 8$, there are $N$ carriers (LOs) with frequencies and phases slightly different from each other. This is represented by the vector $\exp \left(-j 2 \pi\left(F_{c} \underline{1}_{N}+\underline{\widetilde{F}}_{c}\right) t+j \underline{\widetilde{\varphi}}_{c}\right)$ where the vectors $\underline{\widetilde{F}}_{c} \in$ $\mathcal{R}^{N \times 1}$ and $\underline{\tilde{\varphi}}_{c} \in \mathcal{R}^{N \times 1}$ are defined as

$$
\begin{aligned}
\underline{\widetilde{F}}_{c} & =\left[\widetilde{F}_{c_{1}}, \widetilde{F}_{c_{2}}, \cdots, \widetilde{F}_{c_{N}}\right]^{T} \\
\underline{\underline{\varphi}}_{c} & =\left[\widetilde{\varphi}_{c_{1}}, \widetilde{\varphi}_{c_{2}}, \cdots, \widetilde{\varphi}_{c_{N}}\right]^{T}
\end{aligned}
$$

with $\widetilde{F}_{c_{i}}$ and $\widetilde{\varphi}_{c_{i}}$ denote the frequency and phase uncertainties of the LO associated with the $i^{t h}$ array element. In this case, the signal received by the array (point B) is now modelled by

$$
\underline{\widetilde{x}}(t)=\exp \left(-j 2 \pi \underline{\widetilde{F}}_{c} t\right) \odot \exp \left(-j \underline{\widetilde{\varphi}}_{c}\right) \odot \underline{S} \cdot m(t)+\underline{\widetilde{n}}(t)
$$

where $\underline{S}$ is given by Equations (3) and (4) and $\underline{\tilde{n}}(t) \in \mathcal{C}^{N \times 1}$ denotes the baseband noise.

Figure 3 shows the results of a localisation algorithm for an array of 5 antennas in the case where all the antennas have a common carrier (i.e. coherent array). In particular, the array with geometry (in meters) used in Figure 3 given in Equation 9, operates in the presence of 3 uncorrelated sources operating in the near far field of the array with range and azimuth $\left(5 \mathrm{~km}, 60^{\circ}\right),\left(10 \mathrm{~km}, 65^{\circ}\right)$ and $\left(5 \mathrm{~km}, 200^{\circ}\right)$ under SNR $=20 \mathrm{~dB}$ and $L=1000$ snapshots. Using the received signal model presented in Equation 2, the MUSIC algorithm is used in conjunction with Equations 3 and 4 (i.e. Spherical MUSIC). It is clear that the spherical MUSIC works perfectly without the frequency and phase uncertainties in the array model and provides very accurate location estimate.

$$
\mathbf{r}=\left[\begin{array}{ccccc}
0 & -1229 & -2638 & -1229 & 2549 \\
0 & 4797 & 0 & -4797 & -8071 \\
0 & 0 & 0 & 0 & 0
\end{array}\right]
$$

It should be noted that MUSIC cannot be used as a direction finding algorithm but the spherical MUSIC may be used as a source positioning algorithm. In addition, as each array element has its own carrier, frequency and phase uncertainties

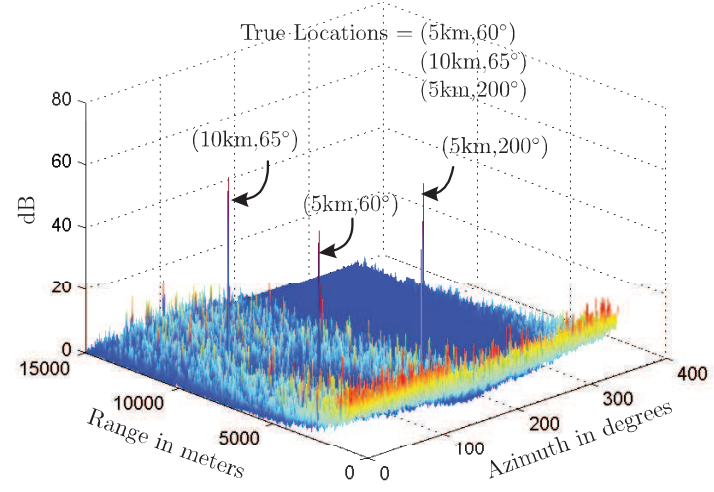

Fig. 3. Performance of the $2 \mathrm{D}$ spherical MUSIC algorithm without frequency and phase uncertainties $L=1000, \mathrm{SNR}=20 \mathrm{~dB}$.

will be the prohibited factor for any algorithm including source localisation using spherical MUSIC.

Using the received signal model of Equation 8, Figure 4 shows the effect of the frequency and phase uncertainties of the local oscillator on the spherical MUSIC algorithm for the same environment used for Figure 3. It is clear from that the spherical MUSIC fails to estimate the location of these sources in the presence of these uncertainties. The aim is therefore to remove these uncertainties by going from Equation 8 to Equation 2 i.e. by tuning the frequencies of each local oscillator to a common frequency.

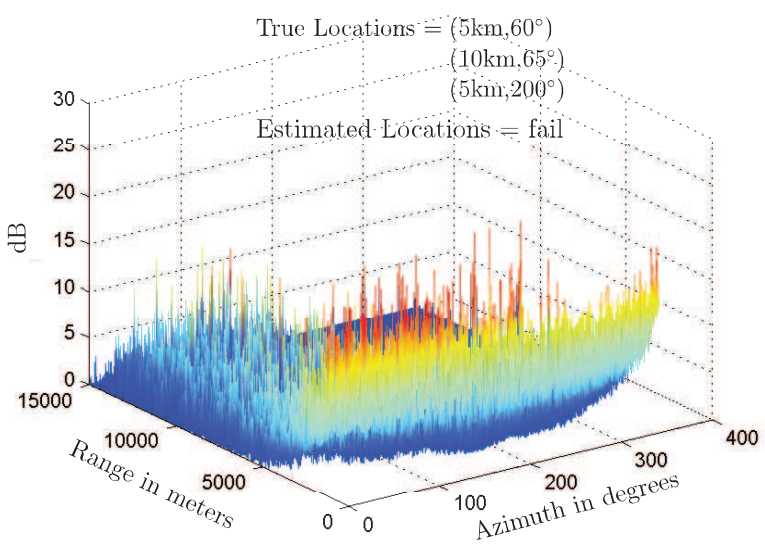

Fig. 4. Performance of the 2D spherical MUSIC algorithm in the presence of frequency and phase uncertainties under $L=1000$ and $\mathrm{SNR}=20 \mathrm{~dB}$.

\section{Removing CARrier UnCERTAinties}

\section{A. Frequency Uncertainty Estimation}

One approach is to use the "harmonic retrieval" modelling in conjunction with a frequency based MUSIC algorithm. Here, the signal received from each array element is sampled with a period $T_{s}$ and passed through a Tapped Delay Line of length $N_{L}$. For the $i^{t h}$ array element, this will create a signal vector 


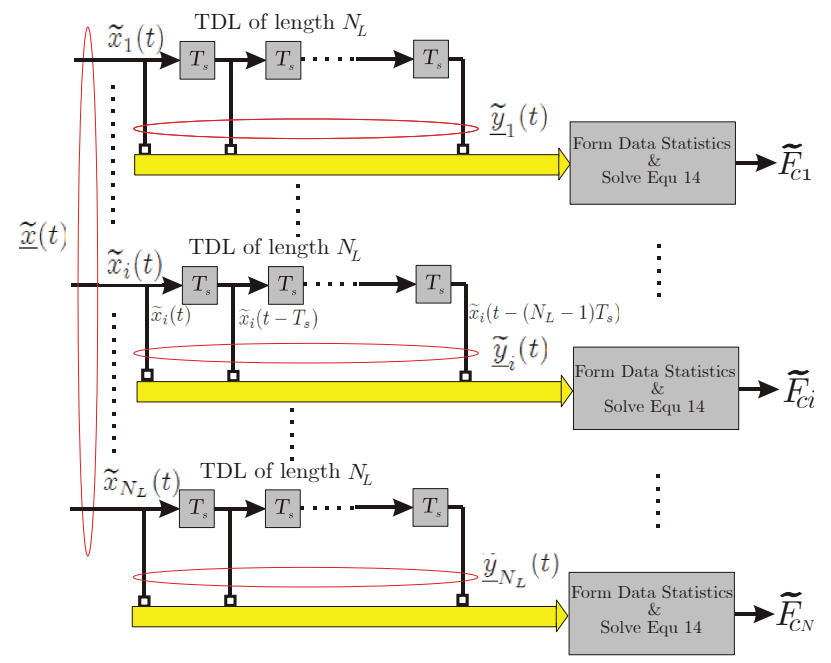

Fig. 5. The harmonic retreival process used to estimate the frequency uncertainties associated with the array elements.

$\underline{y}_{i}(t) \in \mathcal{C}^{N_{L} \times 1}$ as this is shown in Figure 5 whereby

$$
\begin{aligned}
\underline{y}_{i}(t)= & {\left[\widetilde{x}_{i}(t), \widetilde{x}_{i}\left(t-T_{s}\right), \cdots, \widetilde{x}_{i}\left(t-\left(N_{L}-1\right) T_{s}\right)\right]^{T} } \\
= & \underline{A}_{i} m_{i}(t)+\underline{\widetilde{n}}_{i}(t) ; \forall i \in[1, \ldots, N]
\end{aligned}
$$

In Equation 11, the vector $\underline{A}_{i}$ is the temporal manifold of the $i^{\text {th }}$ node defined as

$$
\underline{A}_{i} \triangleq \underline{A}_{i}\left(\widetilde{F}_{c_{i}}\right)=\exp \left(j 2 \pi \widetilde{F}_{c_{i}}\left[0,1, \cdots, N_{L}-1\right]^{T} T_{s}\right)
$$

and

$$
m_{i}(t)=S_{i} \cdot \exp \left(-j \widetilde{\varphi}_{c_{i}}\right)
$$

with $S_{i}$ representing the $i^{\text {th }}$ element of Equation 3. By constructing the covariance matrix, $\mathbb{R}_{i}=\mathcal{E}\left\{\underline{y}(t) \underline{y}(t)^{H}\right\}$, of the vector signal $y_{i}(t)$, the tuning error $\widetilde{F}_{c_{i}}$ can be estimated using for instance, the following MUSIC based estimation problem

$$
\widetilde{F}_{c_{i}}=\arg \min _{\widetilde{f}} \xi_{i}(\widetilde{f}) ; \forall i
$$

where,

$$
\xi_{i}(\widetilde{f})=-10 \log _{10}\left(\underline{A}_{i}(\tilde{f})^{H} \mathbb{E}_{n, i} \mathbb{E}_{n, i}^{H} \underline{A}_{i}(\tilde{f})\right)
$$

with $\mathbb{E}_{n, i}$ denoting the noise subspace eigenvectors of $\mathbb{R}_{i}$. This process should be done in parallel for all the array elements to find the vector $\widetilde{F}_{c}$. Figure 5 illustrates this process.

Following this, the received signal can easily be downconverted to baseband via multiplication. That is

$$
\begin{gathered}
\underline{\hat{x}}(t)=\underline{\widetilde{x}}(t) \odot \exp \left(j 2 \pi \underline{\widetilde{F}}_{c} t\right) \\
\underline{\hat{x}}(t)=\underline{S} \cdot m(t) \odot \exp \left(-j \underline{\widetilde{\varphi}}_{c}\right)+\underbrace{\underbrace{}_{\underline{\widetilde{n}}}(t) \odot \exp \left(j 2 \pi \underline{\widetilde{F}}_{c} t\right)}_{\text {redefined as } \underline{n}(t)}
\end{gathered}
$$

$$
\underline{\hat{x}}(t)=\underline{S} \cdot m(t) \odot \exp \left(-j \underline{\widetilde{\varphi}}_{c}\right)+\underline{n}(t) .
$$

Here note that $\underline{n}(t)$ is the AWGN signal. It is important to note that the frequency uncertainties estimation algorithm is independent of the array reference point. Equation 18 above still contains the phase uncertainties which has to be removed to achieve a fully coherent system.

\section{B. Phase Uncertainty Estimation}

In this method, a pilot source with known location is used, this allows the phase uncertainties associated with the array to be easily estimated using a single transmission. To estimate the phase uncertainties, the covariance matrix of the received baseband signal is constructed and the eigenvector decomposition is performed to extract the eigenvector corresponding to the principle eigenvalue.

$$
\mathcal{L}\left\{\underline{E}_{s}\right\}=\mathcal{L}\left\{\underline{S} \odot \exp \left(-j \underline{\widetilde{\varphi}}_{c}\right)\right\} .
$$

This implies that the eigenvector $\underline{E}_{s}$ and manifold vector $\underline{S}$ spans the same one dimensional subspace, i.e. they are colinear vectors

Hence,

$$
\underline{\widetilde{\varphi}}_{c}=\bmod _{2 \pi}\left(\frac{2 \pi F_{c i}}{c} \underline{\rho}\right)-\measuredangle \underline{E}_{s}
$$

where $\measuredangle$ denotes the element by element angle of a vector and $\underline{\rho}$ represents the range of the transmitter to the $N$ array elements. The steps are summarized below:

\section{STEP-1 Using the downconverted baseband signal in Equa- tion 18 , the covariance matrix is constructed. \\ STEP-2 Extract the eigenvector $E_{s}$ corresponding to the principle eigenvalue. \\ STEP-3 Solve Equation 20 to obtain the estimate of the phase uncertainties. \\ STEP-4 Remove the phase uncertainties by multiplying the baseband signal in Equation 18 with the complex conjugate of the estimated phase.}

By carrying out these aforementioned steps, these phase uncertainties can be estimated and removed from Equation 18, and Equation 2 can easily be obtained.

\section{Simulation Results}

Consider an array of $N=5$ elements forming an arc with very large intersensor spacing operating at $F_{c}=10 \mathrm{MHz}$. The Cartesian coordinates of the array are given by Equation 9 . With reference to Figure 2, the carrier frequency and phase uncertainties are assumed to have the values given in Table I. Figure 6 shows the carrier frequency uncertainties and Table II shows the estimation errors of the phase uncertainties after calibration using the proposed approach.

It is evident that the values in Table II are very small implying that the phase uncertainties are estimated to a high accuracy to provide a fully coherent array system. However, it is important to point out that the presence of phase uncertainties does not affect the frequency estimation algorithm. 
TABLE I

LOCAL OSCILLATOR CHARACTERISTICS

\begin{tabular}{|c|c|c|c|c|c|}
\hline & $\mathrm{Rx} 1$ & $\mathrm{Rx} 2$ & $\mathrm{Rx} 3$ & $\mathrm{Rx} 4$ & $\mathrm{Rx} 5$ \\
\hline \hline$\widetilde{F}_{c}(\mathrm{~Hz})$ & 0 & -200 & -220 & 30 & -20 \\
\hline \hline$\widetilde{\varphi}_{c}\left({ }^{\circ}\right)$ & 0 & 34 & 78 & 29 & 67 \\
\hline \hline
\end{tabular}

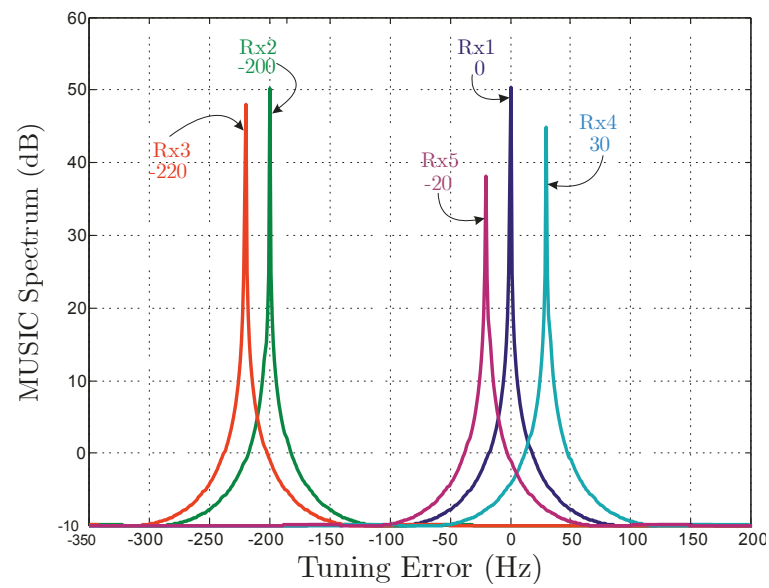

Fig. 6. Representative example of the harmonic retrieval MUSIC approach for the large aperture array under the LO uncertainties in Table I. SNR $=20 \mathrm{~dB}$, $L=1000, N_{L}=10$.

TABLE II

Local Oscillator Phase Estimation after Calibration $\mathrm{SNR}=20 \mathrm{~dB}$ AND $L=1000$

\begin{tabular}{||c||c||c||c||c||c||}
\hline \hline & $\mathrm{Rx} 1$ & $\mathrm{Rx} 2$ & $\mathrm{Rx} 3$ & $\mathrm{Rx} 4$ & $\mathrm{Rx} 5$ \\
\hline \hline$\Delta \widetilde{\varphi}_{c}\left(^{\circ}\right)$ & 0 & 0.0002 & 0.0137 & -0.0167 & -0.010 \\
\hline \hline
\end{tabular}

The performance of the proposed calibration technique was tested with 3 uncorrelated sources operating in the near far field of the array as described in Figure 3. Consider that the desired source is at azimuth $\theta=60^{\circ}$, and the sources at $65^{\circ}$ and $200^{\circ}$ are co-channel interference that must be rejected. A super-resolution beamformer which is designed to maximise the Signal to Interference Ratio (SIR) is employed. Figure 8 shows the array pattern in the presences of these uncertainties described in Table I while Figure 9 shows the array pattern after implementing the proposed algorithm. It is clear from Figure 8 that the gain at the desired signal at $\left(5 \mathrm{~km}, 60^{\circ}\right)$ is $9.71 \mathrm{~dB}$. However, after implementing the proposed calibration technique, Figure 9 shows a gain of $24.11 \mathrm{~dB}$ at the desired signal which is a performance improvement of $14.395 \mathrm{~dB}$. Further, there is a sharper and deeper null at the two interferences after implementing the proposed algorithm as shown in Figure 9. Therefore, the proposed algorithm shows a significant improvement in the SNIR performance of the beamfomer.

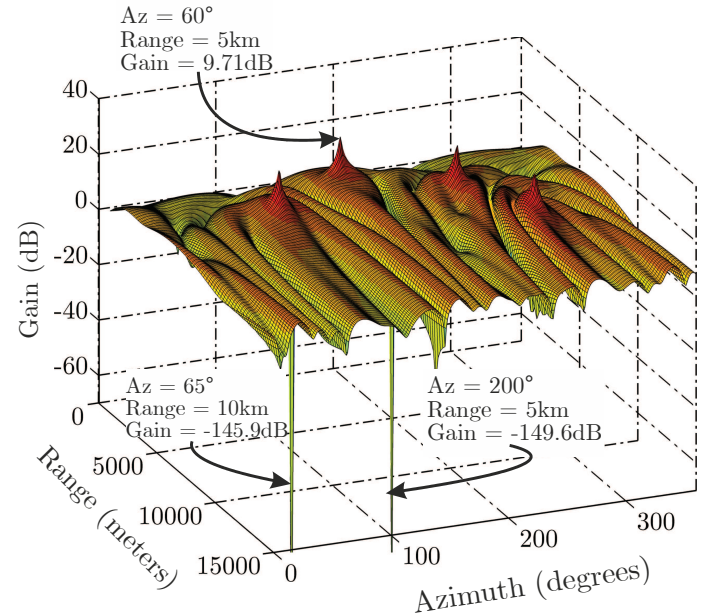

Fig. 8. Array pattern of the Wiener-Hopf beamformer before the proposed calibration approach in the presence of uncertainties described in Table I and geometry in Equation 9. The DOA of the desired source is (range, azimuth) $=\left(5 \mathrm{~km}, 60^{\circ}\right)$ and there two interefences at (range, azimuth $)=\left(10 \mathrm{~km}, 65^{\circ}\right)$ and $\left(5 \mathrm{~km}, 200^{\circ}\right)$. Sources are observed under $L=1000$ snapshot and SNR $=20 \mathrm{~dB}$ (Gain axis has been truncated to $-70 \mathrm{~dB}$ ).

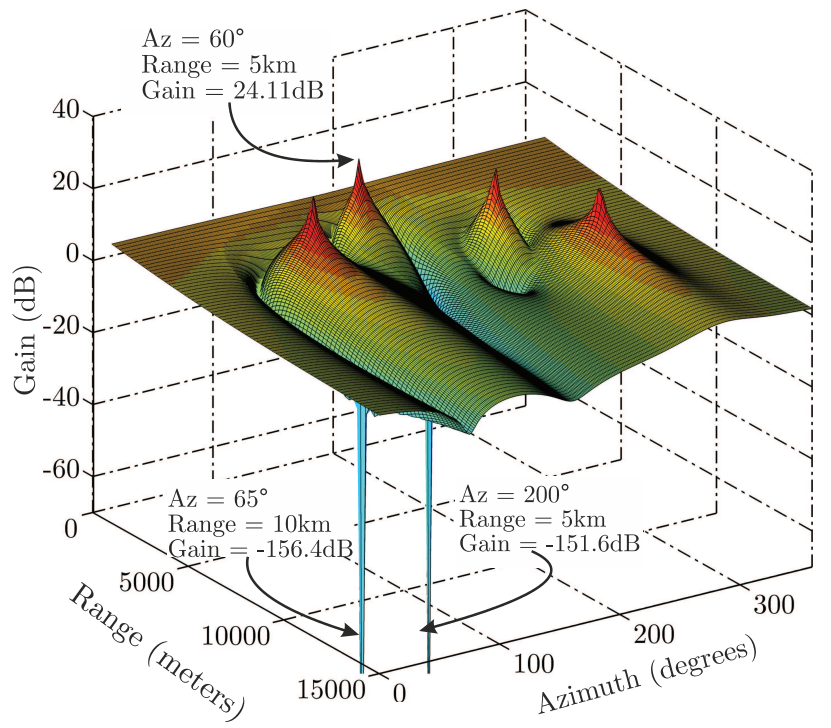

Fig. 9. Array pattern of the Wiener-Hopf beamformer after implementing the proposed calibration approach. The DOA of the desired source is (range, azimuth $)=\left(5 \mathrm{~km}, 60^{\circ}\right)$ and there two interefences at (range, azimuth) $=$ $\left(10 \mathrm{~km}, 65^{\circ}\right)$ and $\left(5 \mathrm{~km}, 200^{\circ}\right)$. Sources are observed under $L=1000$ snapshot and SNR $=20 \mathrm{~dB}$ (Gain axis has been truncated to $-70 \mathrm{~dB}$ ).

\section{EXPERIMENTAL RESUlts Using SDR}

SDR systems have experienced significant proliferation over the last decade as a result of the advances in digital electronics. They provide huge flexibility to designers by allowing them to readily modify and rapidly adapt the system to specific application requirements. These systems provide attractive solutions for testing and deploying wide range of array signal processing algorithms at several transmission bandwidths and frequencies with varying number of sensors. In most applications, the SDR systems have been used as a standalone point-to-point communication device but in this experiment, these SDR units 


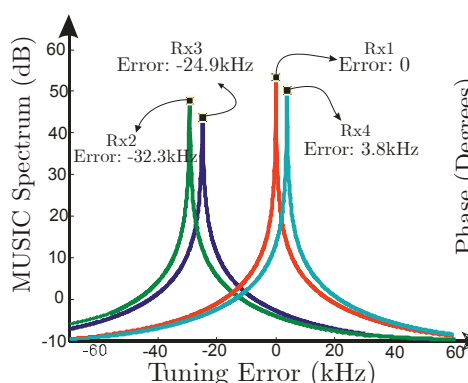

(a) Before Frequency Synchronization

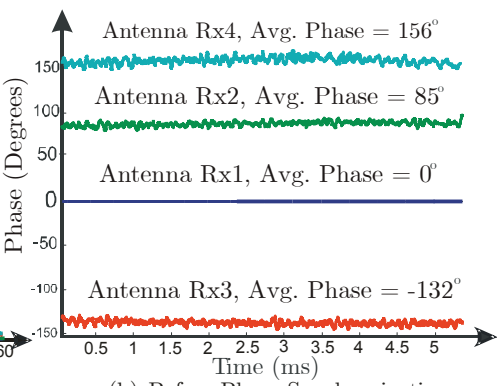

(b) Before Phase Synchronization

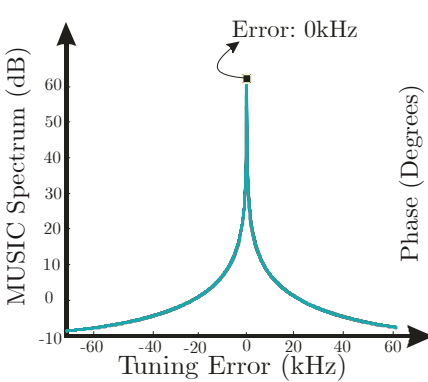

(c) After Frequency Synchronization

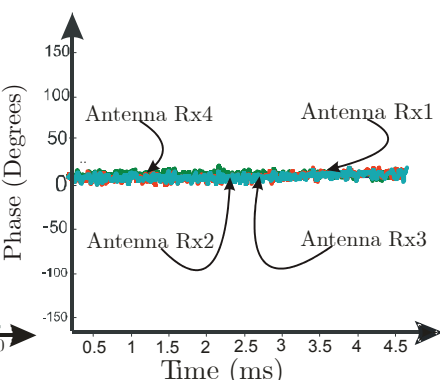

(d) After Phase Synchronization

Fig. 7. Frequency and phase synchronization implementation.

are designed to operate together as an array system. In the experimental setup, four Universal Software Radio Peripheral (USRP2) boards with RFX2400 daughterboards manufactured by Ettus Research operating at $F_{c}=2.43 \mathrm{GHz}$ were used to form an array of $N=4$ sensors. For the array testbed to be considered as fully synchronized, there are three main requirements that must be satisfied. Firstly, the samples from individual receiver must originate from the same sampling clock edge, i.e. the clocks of each of the channels must be synchronized. Secondly, the timestamps of the samples coming from individual receiver chains must be aligned in time. Finally, the frequencies of the individual local oscillators (LOs) must be matched and any phase differences between the LOs must be fixed, known and ideally constant between the hardware power cycles [10]. All the USRP2 boards have two inputs for $10 \mathrm{MHz}$ and $1 \mathrm{~Hz}$ (PPS) external synchronization signals. The $10 \mathrm{MHz}$ input signal is used to align the internal $100 \mathrm{MHz}$ oscillators of each boards while the $1 \mathrm{~Hz}$ PPS signal is used to ensure timestamp alignment of the individual samples (this ensures that the counters of the boards continuously reset at the rising edge of the signal). The LabVIEW software was used to design a virtual instrument (VI) which interfaces with the USRP2 boards to collect data and exported to the MATLAB software for further analysis. Figure 10 shows the hardware configuration setup. It should be noted that for the lab environment, the array geometry has been scaled down by a factor in order to achieve a large aperture array and the transmitter was placed in the vicinity of the array.

Figure 7(a) and 7(b) respectively show the measured frequency and phase errors before synchronization. For instance, it can be seen that in the second antenna (RX2), the errors

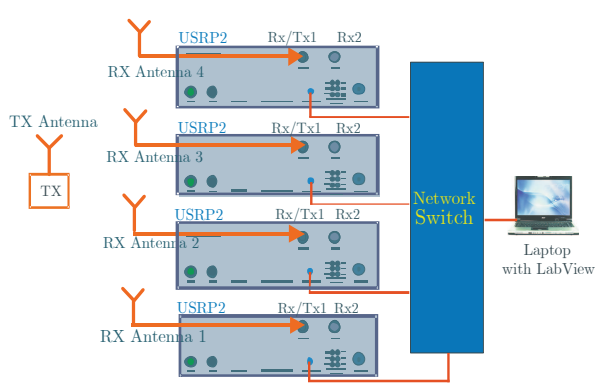

Fig. 10. Array testbed setup using 4 USRP boards. are $-32.3 \mathrm{kHz}$ and $85^{\circ}$. Figure $7(\mathrm{c})$ and $7(\mathrm{~d})$ show when these errors are removed and the array receivers become synchronized.

\section{CONCLUSIONS}

In this paper, a novel algorithm that estimates the frequency and phase uncertainties/errors in a widely distributed array of sensors with independent local oscillator (LO) is proposed. This approach employs harmonic retrieval modelling in conjunction with a frequency based MUSIC algorithm to estimate the frequency uncertainties as well as the phase uncertainties between the array elements. Further, it is shown that the 2D spherical MUSIC source localization algorithm fails in the presence of these uncertainties but performs accurately after implementing the proposed algorithm. Simulation results as well as experimental results are presented.

\section{REFERENCES}

[1] M. Willerton and A. Manikas, "Array Shape Calibration using a Single Multi-Carrier Pilot," in Sensor Signal Processing for Defence, (SSPD), 2011.

[2] A. L. Swindlehurst and T. Kailath, "A Performance Analysis of Subspace-Based Methods in the Presence of Model Errors, Part I : The MUSIC Algorithm," IEEE Transactions on Signal Processing, vol. 40, no. 7, pp. 1758-1774, 1992.

[3] D. Fuhrmann, "Estimation of Sensor Gain and Phase," IEEE Transactions on Signal Processing, vol. 42, no. 1, pp. 77-87, 1994.

[4] N. Fistas and A. Manikas, "A New General Global Array Calibration Method," Proceedings of ICASSP '94. IEEE International Conference on Acoustics, Speech and Signal Processing, vol. iv, no. 1, pp. 73-76, 1994.

[5] A. Manikas and N. Fistas, "Modelling and estimation of mutual coupling between array elements," Proceedings of ICASSP '94. IEEE International Conference on Acoustics, Speech and Signal Processing, vol. iv, pp. IV/553-IV/556, 1994.

[6] K. V. Stavropoulos and A. Manikas, "Array Calibration in the Presence of Unknown Sensor Characteristics and Mutual Coupling," in EUSIPCO Proceedings, 2000, pp. 1417-1420.

[7] G. Efstathopoulos and A. Manikas, "A blind Array Calibration Algorithm Using a Moving Source," in IEEE Sensor Array and Multichannel Signal Processing Workshop SAM 2008., 2008, pp. 455-458.

[8] B. P. Flanagan and K. L. Bell, "Array self-calibration with large sensor position errors," Elsevier Science Signal Processing, vol. 81, no. 10, pp 2201-2214, Oct. 2001.

[9] A. J. Weiss and B. Friedlander, "Array Shape Calibration Using Sources in Unknown Locations- A Maximim Likelihood Approach," IEEE Transactions on Acoustics, Speech, and Signal Processing, vol. 37, no. 12, 1989.

[10] A. Akindoyin, M. Willerton, and A. Manikas, "Localization and Array Shape Estimation Using Software Defined Radio Array Testbed," in IEEE 8th Sensor Array and Multichannel Signal Processing Workshop (SAM), Spain, 2014, pp. 189-192. 